\title{
Force sensing with an optically levitated charged nanoparticle
}

Cite as: Appl. Phys. Lett. 111, 133111 (2017); https://doi.org/10.1063/1.4993555

Submitted: 29 June 2017 . Accepted: 15 September 2017. Published Online: 28 September 2017

David Hempston, Jamie Vovrosh, Marko Toroš (D, George Winstone, Muddassar Rashid, and Hendrik Ulbricht
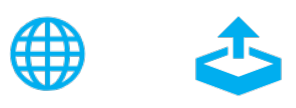

View Online

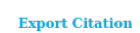

\section{ARTICLES YOU MAY BE INTERESTED IN}

Cooling and manipulation of a levitated nanoparticle with an optical fiber trap

Applied Physics Letters 107, 151102 (2015); https://doi.org/10.1063/1.4933180

Calibration and energy measurement of optically levitated nanoparticle sensors

Review of Scientific Instruments 89, 033111 (2018); https://doi.org/10.1063/1.5017119

Optical trapping and control of nanoparticles inside evacuated hollow core photonic crystal fibers

Applied Physics Letters 108, 221103 (2016); https://doi.org/10.1063/1.4953025

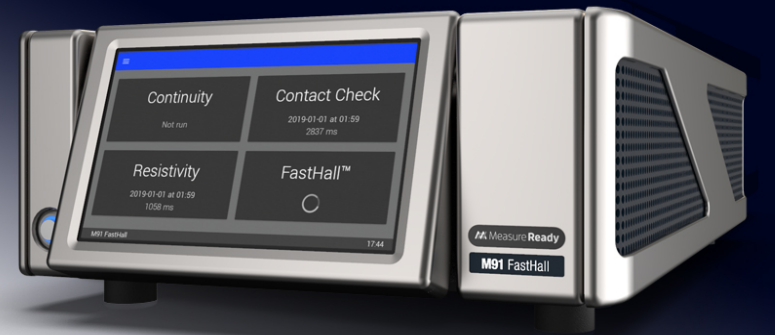

\section{Measure Ready} M91 FastHall ${ }^{\mathrm{TM}}$ Controller

A revolutionary new instrument for complete Hall analysis 


\title{
Force sensing with an optically levitated charged nanoparticle
}

\author{
David Hempston, Jamie Vovrosh, Marko Toroš, George Winstone, Muddassar Rashid, ${ }^{\text {a) }}$ \\ and Hendrik Ulbricht ${ }^{\text {b) }}$ \\ Department of Physics and Astronomy, University of Southampton, Southampton SO17 1BJ, United Kingdom
}

(Received 29 June 2017; accepted 15 September 2017; published online 28 September 2017)

\begin{abstract}
Levitated optomechanics is showing potential for precise force measurements. Here, we report a case study to show experimentally the capacity of such a force sensor, using an electric field as a tool to detect a Coulomb force applied onto a levitated nanosphere. We experimentally observe the spatial displacement of up to $6.6 \mathrm{~nm}$ of the levitated nanosphere by imposing a DC field. We further apply an $\mathrm{AC}$ field and demonstrate resonant enhancement of force sensing when a driving frequency, $\omega_{A C}$, and the frequency of the levitated mechanical oscillator, $\omega_{0}$, converge. We directly measure a force of $3.0 \pm 1.5 \times 10^{-20} \mathrm{~N}$ with $10 \mathrm{~s}$ integration time, at a centre of mass temperature of $3 \mathrm{~K}$ and at a pressure of $1.6 \times 10^{-5}$ mbar. Published by AIP Publishing.

https://doi.org/10.1063/1.4993555
\end{abstract}

The ability to detect forces with increasing sensitivity is of paramount importance in many fields of study, from detecting gravitational waves ${ }^{1}$ to molecular force microscopy of cell structures and their dynamics. ${ }^{2}$ In the case of a mechanical oscillator, the force sensitivity limit arises from the classical thermal noise, as given by

$$
S_{F F}^{\text {th }}=\sqrt{4 k_{b} T m \omega_{0} / Q_{m}},
$$

where $k_{b}$ is the Boltzmann constant, $T$ is the temperature of the thermal environment, $m$ is the mass of the object, $\omega_{0}$ is the oscillator angular frequency, $Q_{m}=\omega_{0} / \Gamma_{0}$ is the mechanical quality factor, and $\Gamma_{0}$ is the damping factor. In recent decades, systems, such as cold-atoms traps, have pushed the boundaries of force sensitivities down to $1 \times 10^{-24} \mathrm{~N} / \sqrt{\mathrm{Hz}}{ }^{3}$ whilst trapped-ions have demonstrated force sensitivities below $500 \times 10^{-24} \mathrm{~N} / \sqrt{\mathrm{Hz}},{ }^{4}$ with prospects of even lower force sensitivities with novel geometries. ${ }^{5}$ On a more macroscopic level, cantilever devices are able to achieve force sensitivities, reportedly down to $10^{-21} \mathrm{~N} / \sqrt{\mathrm{Hz}}$ and $Q_{m^{-}}$ factors of greater than one million. ${ }^{6-13}$ Parallel to cantilever devices, toroidal microresonators have achieved modest levels of force sensitivities $\sim 1 \times 10^{-18} \mathrm{~N} / \sqrt{\mathrm{Hz}}{ }^{14}$ Such toroidal microresonators have achieved $Q_{m}$-factors of up to $10^{9},{ }^{15}$ and position sensitivities down to $1 \times 10^{-19} \mathrm{~m} / \sqrt{\mathrm{Hz}} .{ }^{16}$ These devices have a number of applications, especially as on-chip force transducers. ${ }^{17}$ However, such devices are strongly limited by noise due to mechanical coupling to the environment.

The fundamental requirements for a good force sensor are [according to Eq. (1)] good mechanical isolation from external noise or a high $Q_{m}$-factor, low environmental temperatures, and ideally low oscillation frequencies.

In levitated optomechanics, focused light is used for trapping particles in air and vacuum. ${ }^{18}$ Levitated particles are more isolated mechanically from their environment than clamped systems and exhibit high $Q_{m}$-factors of greater than $10^{6}$ (Refs. 19-21) in translational motion, which, in principle, are limited only by the background gas pressure and thus are predicted to reach $Q_{m}$ factors $>10^{12}$. Recently, Kuhn et al. ${ }^{22}$ have reached $Q_{m}$ of up to $10^{11}$ for a driven rotational degree of motion of a levitated nanorod at a few millibars of pressure, at room temperature. Translational motion, generally, is calculated to have force sensitivities of $1 \times 10^{-21} \mathrm{~N} / \sqrt{\mathrm{Hz}},{ }^{23}$ whilst rotational or torsional degrees of freedom of a trapped non-spherical nanoparticle are predicted to have torsional force sensitivities of $2.4 \times 10^{-22} \mathrm{Nm} /$ $\sqrt{\mathrm{Hz}}$ (Ref. 22) to $2 \times 10^{-29} \mathrm{Nm} / \sqrt{\mathrm{Hz}} .{ }^{24}$ As a consequence of these prospects, levitated optomechanics has attracted interest for precision measurements in electron spin resonance, ${ }^{25,26}$ short-range forces,${ }^{27}$ high-frequency gravitational waves, ${ }^{28}$ tests of collapse models, ${ }^{29,30}$ the SchrödingerNewton equation, ${ }^{31}$ and direct dark matter detection. ${ }^{32}$

Charged levitated particles have been studied earlier in a hybrid optical-electric Paul trap, ${ }^{33}$ in the search for millicharges $^{34}$ as well as for the demonstration of charge control in nanoparticles. ${ }^{3,35}$ The control of charges on nanoparticles is essential for experiments to prepare non-classical states of motion of the particle. ${ }^{30,36}$ In addition, force detection at $1.63 \times 10^{-18} \mathrm{~N} / \sqrt{\mathrm{Hz}}$ in levitated nanospheres has already been demonstrated ${ }^{20}$ by experiments.

Here, we take a detailed look at the interaction of an optically levitated dielectric charged particle with an external electric field as a case study for force sensing. We measure the effect of the Coulomb interaction on the motion of a single nanoparticle, at high vacuum $\left(10^{-5} \mathrm{mbar}\right)$ by applying a DC and an AC electric field to a metallic needle positioned near the trapped particle. These particles can carry multiple elementary electric charges $\left(e=1.6 \times 10^{-19} \mathrm{C}\right)$, and we use the Coulomb interaction to determine the number of elementary charges attached to the particle.

The charge at the needle tip, $q_{t}$, for a given applied voltage is, according to Gauss's Law, $\oint_{s} \mathbf{E} \cdot d \mathbf{s}_{\mathbf{t}}=\frac{q_{t}}{\epsilon_{0}}$, where $\mathbf{s}_{\mathbf{t}}$ is the surface of the needle tip, $\epsilon_{0}$ is the vacuum permittivity, and $\mathbf{E}$ is the electric field. The electric field at any point in a potential, $V$, is given by $-\nabla V=\mathbf{E}$. If we approximate the needle tip as a sphere of radius, $r_{t}$, then $d \mathbf{s}_{\mathbf{t}}=4 \pi r_{t} d \mathbf{r}$. We get

$$
\oint r_{t} \frac{d v}{d r} 4 \pi r_{t} d r=4 \pi r_{t} V=\frac{q_{t}}{\epsilon_{0}} .
$$

\footnotetext{
${ }^{\text {a)m.rashid@soton.ac.uk }}$
} 
Combining this with the Coulomb force acting on the particle at distance $d$ we get

$$
F_{C}=\frac{q_{p} q_{t}}{4 \pi \epsilon_{0} d^{2}}=\frac{q_{p} V r_{t}}{d^{2}},
$$

where $q_{p}$ is the charge on the nanoparticle. This additional force displaces the optically trapped particle. With Eq. (3), the nanoparticles' equation of motion can be written as

$$
\ddot{x}(t)+\Gamma_{0} \dot{x}(t)+\frac{k}{m} x(t)=\frac{F_{t h}(t)}{m}+\frac{F_{C}}{m} e^{i \omega_{A C} t},
$$

where $k$ is the spring constant according to the optical gradient force on the particle, and $F_{t h}$ is a stochastic force originated by a random process that satisfies the fluctuation-dissipation theorem. ${ }^{37}$ The AC driving frequency, $\omega_{A C}$, is zero when considering the DC case. The time averaged position is now nonzero relative to the trap centre and is given by

$$
k_{z}\langle z\rangle=\cos (\theta) \frac{q V r_{t}}{\langle d\rangle^{2}},
$$

where $\langle z\rangle$ is the time average of the position in $z$ direction, and $\theta$ is the angle between the direction of the force and the $z$ direction. $d$ is the distance between the needle and the trapped particle; thus, we can write $\langle z\rangle=d^{\prime}-\langle d\rangle$, where $d^{\prime}$ is the distance between the centre of the laser focus and needle tip. Taking $\langle z\rangle^{2}$ to be small in the resulting quadratic equation and noting that $k_{z}=\omega_{z}^{2} m$, we get the new average position to be

$$
\langle z\rangle=\cos (\theta) \frac{q V r_{t}}{\omega_{z}^{2} m d^{\prime 2}} .
$$

In the case of $\mathrm{AC}, \omega_{A C} \neq 0$, and thus, the $\mathrm{AC}$ contribution in the equation of motion in Eq. (4) has to be considered. Here, we would like to look at the particles' motion at the driving frequency

$$
z(t)=z_{0} e^{i \omega_{A C} t}
$$

Inserting Eq. (7) into Eq. (4) and multiplying by the complex conjugate gives the peak height of the particles motion at $\omega_{A C}$

$$
S_{A C}\left(\omega_{A C}\right)=\frac{1}{m^{2}} \frac{\left|F_{t h}\right|^{2}+\left|F_{C}\right|^{2}}{\left(\omega_{0}^{2}-\omega_{A C}^{2}\right)^{2}+\left(\Gamma_{0}+\delta \Gamma\right)^{2} \omega_{A C}^{2}},
$$

where $\left|F_{t h}\right|^{2}=k_{B} T 2 m \Gamma_{0} / \pi$ and $\delta \Gamma$ is the additional damping due to the parametric feedback. The analysis above demonstrates that the DC contribution results in a shift in the average position of the trap, whilst the $\mathrm{AC}$ driving introduces resonance enhancement of the amplitude of the oscillation signal. Without additional forces, the Power Spectral Density (PSD) of the particle's motion is given by

$$
S_{x x}(\omega)=\gamma^{2} \frac{k_{B} T_{0}}{\pi m} \frac{\Gamma_{0}}{\left(\omega_{0}^{2}-\omega^{2}\right)^{2}+\Gamma_{0}^{2} \omega^{2}},
$$

where $\gamma$ is the conversion factor that converts the detection voltage to metres. ${ }^{21}$ By fitting Eq. (9) to the experimentally measured PSD [see Fig. 1(a)], the damping from background gas $\Gamma_{0}$ and feedback cooling $\delta \Gamma$ can be determined. These fitted parameters can then be used to work out the radius and mass of the particle, as well as the centre-of-mass (c.m) temperature of the trapped particle from $T_{c m}=\frac{T_{0} \Gamma_{0}}{\Gamma_{0}+\delta \Gamma}$.

In our experiments, we trap a silica nanoparticle (density, $\rho_{\mathrm{SiO}_{2}} \sim 2.65 \mathrm{~g} / \mathrm{cm}^{3}$ ) in a dipole trap. The optical gradient force trap is realised using a $1550 \mathrm{~nm}$ laser and a high numerical aperture (N.A.) parabolic mirror to produce a diffraction limited focus. The particle's position is measured by detecting the interference between the light Rayleigh scattered by the particle and the divergent reference light with a single photodiode [as shown in Fig. 1(b)]. The detected signal contains three distinct frequencies for motion along $x, y$, and $z$ directions, each of which is sent to a lock-in amplifier. The amplifiers output to an acoustic optical modulator (AOM) at twice the trap frequency with an appropriate phase shift that counters the $c . m$ motion of the particle, thus cooling the $c . m$ temperature. More details can be found elsewhere. ${ }^{21}$ For both DC and AC cases, we carry out the experiments at a pressure of $1.6 \times 10^{-5}$ mbar and we cool the particle motion to $\sim 3 \mathrm{~K}$ in the $z$-axis [see Fig. 1(a)]. The needle that is used to generate the DC/AC electric field is made of polished stainless steel and has a tip radius of $100 \mu \mathrm{m}$. The distance between the trap centre to the needle, $d^{\prime}$, is measured to be $39.6 \pm 0.8 \mathrm{~mm}$ and $\theta=45^{\circ}$. To generate the DC field, we connected the needle to a high power supply (Berta High Voltage Power Supply
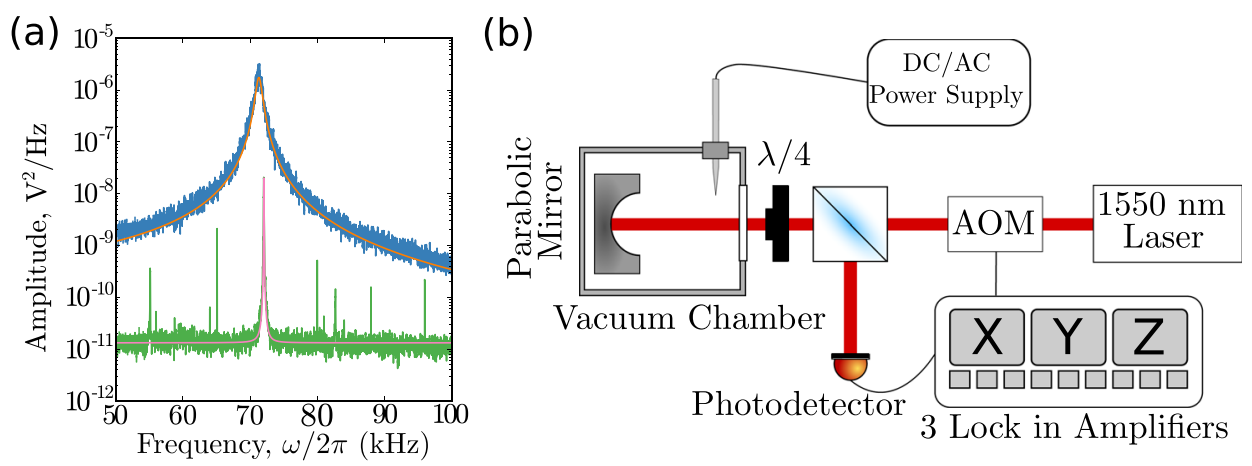

FIG. 1. Power spectral density and experimental setup: (a) Cooling the motion in z-direction of a $41 \pm 6 \mathrm{~nm}$ radius particle, the upper spectrum (blue) is at $3 \pm 0.3 \mathrm{mbar}$ and the lower spectrum is at $4.5 \times 10^{-5} \mathrm{mbar}$. This corresponds to a temperature of $\sim 3 \mathrm{~K}$ from $300 \mathrm{~K}$. (b) A needle is connected via a high voltage vacuum feedthrough to either a DC power supply that can output up to $20 \mathrm{kV}$ or a signal generator for the AC experiments. The distance from the needle tip to the laser focus $d^{\prime}$ is measured to be $39.6 \pm 0.8 \mathrm{~mm}$ and at $\theta=45^{\circ}$. The mirror, along with the whole chamber, is earthed. 
230 series), and to generate the $\mathrm{AC}$ field, we connect to a signal generator (TTi TG1010A Programmable Function Generator).

To study the effect of the DC field, we levitate a $41 \pm 6 \mathrm{~nm}$ (mass, $m=7.6 \times 10^{-19} \mathrm{~kg}$ ) silica particle. The DC field generated by the needle tip modifies the effective potential experienced by the particle. This modification leads to a shift in the mean position of the particle. The spatial displacement is shown in Fig. 2(b) and it increases with increasing DC voltage. Figure 2(a) shows the displacement for particle of charge of $9 e \pm 1 e$ and a spatial displacement of $6.6 \mathrm{~nm}$ for a $V_{d c}=10 \mathrm{kV}$. The displacement operation increases the c.m temperature of the ensemble. The related heating can be explained by increasing the absolute noise on the DC voltage. For small displacements, such as those observed in the present study, the trap stays harmonic. For voltages greater than $10 \mathrm{kV}$, we often lose particles from the trap.

In the case of the AC field, it is apparent from Eq. (8) that when $\omega_{A C}$ is far from $\omega_{0}$ then the PSD signal is weak; however, as the two converge, there is a strong signal enhancement allowing much smaller forces to be detected. Figure 3 shows the peak heights, both for the theory and experimental plots, demonstrating this enhancement effect for a particle of radius $50 \pm 6 \mathrm{~nm}$. Using a pure sine wave as the driving frequency, the detuning, $\Delta \omega=\left(\omega_{0}-\omega_{A C}\right)$, is swept in increments of $500 \mathrm{~Hz}$ across $\omega_{0}$. By fitting the recorded signal amplitude of the driving field in Fig. 3 with Eq. (8), we obtain $F_{A C}$, which we measure, for $1 \mathrm{~V}$ amplitude

(a)

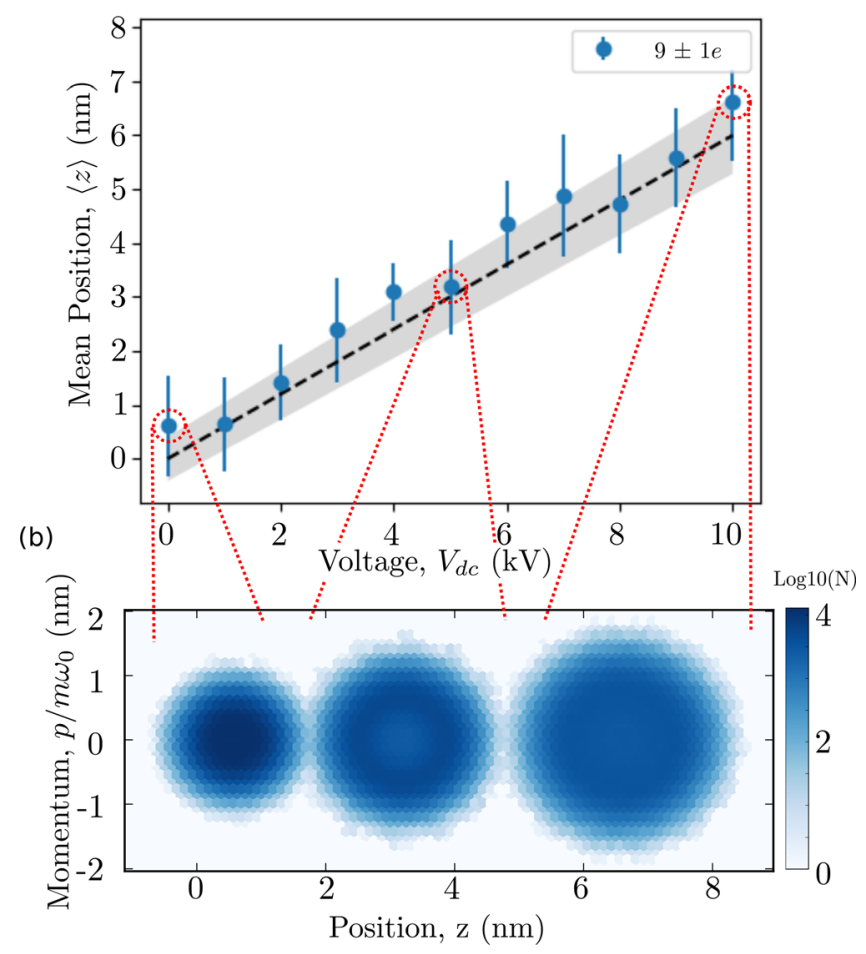

FIG. 2. Spatial displacement: Data show the spatial displacement in the $z$-direction for a particle of radius of $41 \pm 6 \mathrm{~nm}$ for applied DC voltages of $0-10 \mathrm{kV}$. (a) Displacement of the particle's mean position at the application of different DC fields produced by the needle. (b) The displacement of the thermal state distribution at 0,5 , and $10 \mathrm{kV}$ to be $0.6 \mathrm{~nm} 3.1 \mathrm{~nm}$, and $6.6 \mathrm{~nm}$, respectively. Using Eq. (6) gives a charge of $9 \pm 1 e$. Throughout these experiments, the particles $c . m$ temperature is at $\sim 3 \mathrm{~K}$.

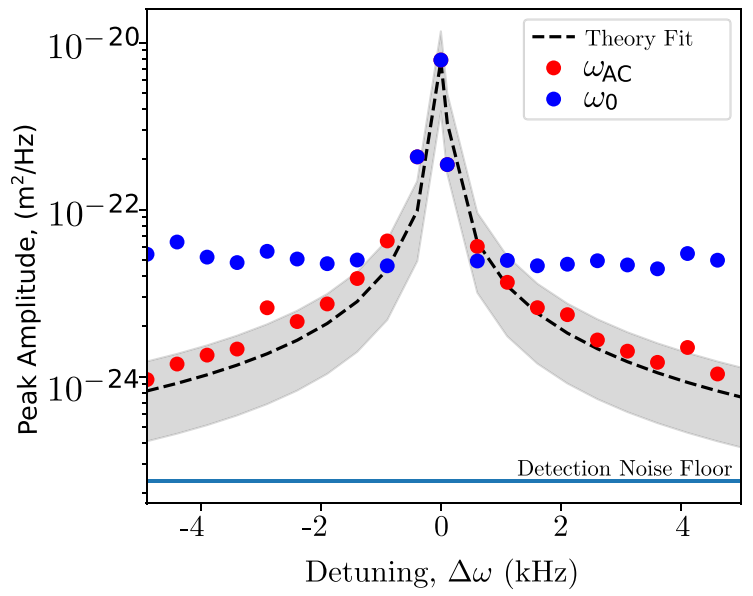

FIG. 3. AC modulation: Peak height of the driving AC field (in red) and levitated oscillator (in blue) frequencies. Equation (8) is fitted to the driving amplitude, and the shaded region is the fitting error. The detuning, $\Delta \omega=\omega_{0}-\omega_{A C}$, is swept from low to high frequencies in steps of $500 \mathrm{~Hz}$ to show the full spectrum response. The averaged experimental noise floor is shown at $1.6 \times 10^{-5}$ mbar. An AC force of $3.0 \pm 1.5 \times 10^{-20} \mathrm{~N}$ and a thermal force of $3.2 \times 10^{-20} \mathrm{~N}$ were measured with $10 \mathrm{~s}$ integration time. The particle has a radius of $50 \pm 6 \mathrm{~nm}$ giving a mass of $1.4 \times 10^{-18} \mathrm{~kg}$ and a charge of $4 \pm 3 e$.

of the AC field on resonance, to be $3.0 \pm 1.5 \times 10^{-20} \mathrm{~N}$ integrated over $10 \mathrm{~s}$. This approaches a force sensitivity of 3.2 $\times 10^{-20} \mathrm{~N} / \sqrt{\mathrm{Hz}}$, which is limited by gas collisions at the pressure in the vacuum chamber. Since, we obtain $F_{A C}$ experimentally, we can relate this to the number of charges on the particle as

$$
F_{A C}=\frac{q_{p} V r_{t}}{d^{2}} .
$$

Thus, the number of elementary charges on the nanoparticle, in the AC experiment, is calculated to be $4 \pm 3 e$. The resonant driven signal is enhanced by a factor of 200 compared to the undriven system.

The limiting factor to reach even lower force sensitivities than $10^{-20} \mathrm{~N} / \sqrt{\mathrm{Hz}}$ can be associated with noise in the present system. In general, this noise is a summation of detector noise, electronic noise via the feedback system, mechanical noise in the optical elements, classical thermal noise due to gas collisions, and the standard quantum limit (SQL). The dominating noise for the current system is the thermal noise floor according to background gas collisions at a pressure of $10^{-5}$ mbar. In addition, long term laser power drifts of approximately $1 \%$, at timescales of hours, are observed. This is due to thermal drifts in the fibre optics which consequently causes a change in polarisation, which affects the trapping power and thus introduces drifts in the trapping frequencies. This power drift predominantly affects the DC experiments, which requires the measurement of many different DC voltages and takes many hours to carry out. In addition, at short time scales, we also have electronic noise due to the feedback system. ${ }^{21}$ The corresponding averaged experimental noise floor is shown Fig. 3. The error bars on the particle size, mass, and charge are dominated by the uncertainty in the pressure readings, which is accurate to $15 \%$.

The classical thermal noise, which has already been discussed in Eq. (1) and for levitated systems is physically 
realised by gas collisions, puts a strong limit on the systems sensitivity. But with modification to the current setup, i.e., for lower pressure $\left(\sim 10^{-9}\right.$ mbar $)$ and with a smaller particle $(\mathrm{r} \sim 10 \mathrm{~nm})$ and trapping frequencies of $\sim 100 \mathrm{kHz}$, force sensitivities down to $1 \times 10^{-24} \mathrm{~N} / \sqrt{\mathrm{Hz}}$ can be reached.

At this limit of $1 \times 10^{-24} \mathrm{~N} / \sqrt{\mathrm{Hz}}$, it is envisaged that the standard quantum limit (SQL) for the system would be reached. The SQL, which can be written as $S_{F F}^{S Q L}$ $=\sqrt{\hbar \omega_{0} m / 2 \tau_{F}}$, where $\tau_{F}$ is the rate of the measurement carried out on the particle ${ }^{38}$ and is calculated for the current system to be $6 \times 10^{-24} \mathrm{~N} / \sqrt{\mathrm{Hz}}$.

In conclusion, we have measured the response of an optically levitated charged nanoparticle to a DC and an AC electric field. We have observed spatial displacement of the centre of the thermal motional state of the particle in phase space by up to $6.6 \mathrm{~nm}$ for an applied DC field of $10 \mathrm{kV}$. We find that by applying an AC field amplitude of $1 \mathrm{~V}$ on resonance we are able to measure a force of $3.0 \times 10^{-20} \mathrm{~N}$. The sensitivity can be, in future experiments, improved by lowering the noise floor, which is limited by the thermal noise of gas collisions at $10^{-5}$ mbar. We extrapolate that by optimising particle size, pressure, and mechanical frequency, we can reach SQL. Then, techniques such as position or momentum squeezing of mechanical oscillators ${ }^{39-41}$ may be used to increase for sensitivities even further. While this gives a direct perspective for the use of levitated optomechanics for force sensing applications, the system is also suitable for fundamental physics problems. The experiment can be used for a non-interferometric test of the quantum superposition principle. ${ }^{29}$ Specifically, the continuous spontaneous localization (CSL) model, ${ }^{42}$ which gives a quantitative violation of the superposition principle, predicts a slight increase in temperature of the trapped nanoparticle. This effect, as discussed in Refs. 43 and 44 , can be used to set bounds ${ }^{45}$ on the CSL parameters, namely, on the localization rate $\lambda$ and on the localization length $r_{C}$. The minimum value of $\lambda$ that could be excluded by the current experimental setup is $\approx 10^{-6} \mathrm{~s}^{-1}$ (achieved at $r_{C} \approx 0.3 \times 10^{-7} \mathrm{~m}$ ), which corresponds to a macroscopicity measure ${ }^{46}$ of $\mu \approx 12$. Increasing the size of the trapped particle to $R=300 \mathrm{~nm}$, which can be trapped by the current experimental setup, would improve the bounds on $\lambda$ by two orders of magnitude.

We thank Phil Connell and Gareth Savage for expert technical help during the realisation of the experimental setup. We would also like to thank Chris Timberlake, Markus Rademacher, and Ashley Setter for the QPlots package and discussions. We also thank The Leverhulme Trust and the Foundational Questions Institute (FQXi) for funding this project.

${ }^{1}$ B. P. Abbott, R. Abbott, T. D. Abbott, M. R. Abernathy, F. Acernese, K. Ackley, C. Adams, T. Adams, P. Addesso, R. X. Adhikari et al., Phys. Rev. Lett. 116, 061102 (2016).

${ }^{2}$ M. Goktas and K. G. Blank, Adv. Mater. Interfaces 4, 1600441 (2017).

${ }^{3}$ M. Gierling, P. Schneeweiss, G. Visanescu, P. Federsel, M. Häffner, D. P. Kern, T. E. Judd, A. Günther, and J. Fortágh, Nat. Nanotechnol. 6, 446 (2011).

${ }^{4}$ M. J. Biercuk, H. Uys, J. W. Britton, A. P. VanDevender, and J. J. Bollinger, Nat. Nanotechnol. 5, 646 (2010).

${ }^{5}$ R. Maiwald, D. Leibfried, J. Britton, J. C. Bergquist, G. Leuchs, and D. J. Wineland, Nat. Phys. 5, 551 (2009).
${ }^{6}$ K. Yasumura, T. Stowe, E. Chow, T. Pfafman, T. Kenny, B. Stipe, and D. Rugar, J. Microelectromech. Syst. 9, 117 (2000).

${ }^{7}$ H. J. Mamin and D. Rugar, Appl. Phys. Lett. 79, 3358 (2001).

${ }^{8}$ D. Rugar, R. Budakian, H. J. Mamin, and B. W. Chui, Nature 430, 329 (2004).

${ }^{9}$ O. Arcizet, P.-F. Cohadon, T. Briant, M. Pinard, A. Heidmann, J.-M. Mackowski, C. Michel, L. Pinard, O. Français, and L. Rousseau, Phys. Rev. Lett. 97, 133601 (2006).

${ }^{10}$ Y. Tao, J. M. Boss, B. A. Moores, C. L. Degen, and S. J. Sharp, Nat. Commun. 5, 3638 (2014).

${ }^{11}$ M. Li, H. X. Tang, and M. L. Roukes, Nat. Nanotechnol. 2, 114 (2007).

${ }^{12}$ J. Moser, A. Eichler, J. Güttinger, M. I. Dykman, and A. Bachtold, Nat. Nanotechnol. 9, 1007 (2014).

${ }^{13}$ P. Weber, J. Güttinger, A. Noury, J. Vergara-Cruz, and A. Bachtold, Nat. Commun. 7, 12496 (2016).

${ }^{14}$ E. Gavartin, P. Verlot, and T. J. Kippenberg, Nat. Nanotechnol. 7, 509 (2012).

${ }^{15}$ M. Goryachev, D. L. Creedon, E. N. Ivanov, S. Galliou, R. Bourquin, and M. E. Tobar, Appl. Phys. Lett. 100, 243504 (2012).

${ }^{16}$ A. Schliesser, G. Anetsberger, R. Rivière, O. Arcizet, and T. J. Kippenberg, New J. Phys. 10, 095015 (2008).

${ }^{17}$ Y.-W. Hu, Y.-F. Xiao, Y.-C. Liu, and Q. Gong, Front. Phys. 8, 475 (2013).

${ }^{18}$ J. M. Ashkin and A. Dziedzic, Appl. Phys. Lett. 28, 333 (1976).

${ }^{19}$ J. Gieseler, B. Deutsch, R. Quidant, and L. Novotny, Phys. Rev. Lett. 109, 103603 (2012).

${ }^{20}$ G. Ranjit, M. Cunningham, K. Casey, and A. A. Geraci, Phys. Rev. A 93, 053801 (2016).

${ }^{21}$ J. Vovrosh, M. Rashid, D. Hempston, J. Bateman, M. Paternostro, and H. Ulbricht, J. Opt. Soc. Am. B 34, 1421 (2017).

${ }^{22}$ S. Kuhn, B. A. Stickler, A. Kosloff, F. Patolsky, K. Hornberger, M. Arndt, and J. Millen, e-print arXiv:1702.07565 (2017).

${ }^{23}$ J. Gieseler, L. Novotny, and R. Quidant, Nat. Phys. 9, 806 (2013).

${ }^{24}$ T. M. Hoang, Y. Ma, J. Ahn, J. Bang, F. Robicheaux, Z.-Q. Yin, and T. Li, Phys. Rev. Lett. 117, 123604 (2016).

${ }^{25}$ T. M. Hoang, J. Ahn, J. Bang, and T. Li, Nat. Commun. 7, 12250 (2016).

${ }^{26}$ L. P. Neukirch, J. Gieseler, R. Quidant, L. Novotny, and A. Nick Vamivakas, Opt. Lett. 38, 2976 (2013).

${ }^{27}$ A. A. Geraci, S. B. Papp, and J. Kitching, Phys. Rev. Lett. 105, 101101 (2010).

${ }^{28}$ A. Arvanitaki and A. A. Geraci, Phys. Rev. Lett. 110, 071105 (2013).

${ }^{29}$ A. Bassi, K. Lochan, S. Satin, T. P. Singh, and H. Ulbricht, Rev. Mod. Phys. 85, 471 (2013).

${ }^{30}$ J. Bateman, S. Nimmrichter, K. Hornberger, and H. Ulbricht, Nat. Commun. 5, 4788 (2014).

${ }^{31}$ A. Großardt, J. Bateman, H. Ulbricht, and A. Bassi, Phys. Rev. D 93, 096003 (2016).

${ }^{32}$ J. Bateman, I. McHardy, A. Merle, T. R. Morris, and H. Ulbricht, Sci. Rep. 5, 8058 (2015).

${ }^{33}$ J. Millen, P. Fonseca, T. Mavrogordatos, T. Monteiro, and P. Barker, Phys. Rev. Lett. 114, 123602 (2015).

${ }^{34}$ D. C. Moore, A. D. Rider, and G. Gratta, Phys. Rev. Lett. 113, 251801 (2014).

${ }^{35}$ M. Frimmer, J. Gieseler, and L. Novotny, Phys. Rev. Lett. 117, 163601 (2016).

${ }^{36}$ O. Romero-Isart, Phys. Rev. A 84, 052121 (2011).

${ }^{37}$ R. Kubo, Rep. Prog. Phys. 29, 255 (1966).

${ }^{38}$ V. Braginsky, F. Khalili, and K. Thorne, Quantum Measurement (Cambridge University Press, 1995).

${ }^{39}$ E. E. Wollman, C. U. Lei, A. J. Weinstein, J. Suh, A. Kronwald, F. Marquardt, A. A. Clerk, and K. C. Schwab, Science 349, 952 (2015).

${ }^{40}$ M. Rashid, T. Tufarelli, J. Bateman, J. Vovrosh, D. Hempston, M. S. Kim, and H. Ulbricht, Phys. Rev. Lett. 117, 273601 (2016).

${ }^{41}$ R. Riedinger, S. Hong, R. A. Norte, J. A. Slater, J. Shang, A. G. Krause, V. Anant, M. Aspelmeyer, and S. Gröblacher, Nature 530, 313 (2016).

${ }^{42}$ G. C. Ghirardi, P. Pearle, and A. Rimini, Phys. Rev. Lett. A 42, 78-89 (1990).

${ }^{43}$ A. Vinante, R. Mezzena, P. Falferi, M. Carlesso, and A. Bassi, Phys. Rev. Lett. 119, 110401 (2017).

${ }^{44}$ A. Vinante, M. Bahrami, A. Bassi, O. Usenko, G. Wijts, and T. Oosterkamp, Phys. Rev. Lett. 116, 090402 (2016).

${ }^{45}$ The ability of the experimental setup to set bounds on the CSL parameters is related to the precision $\Delta T$ with which we can measure the temperature $\mathrm{T}$ of the system. Specifically, we have estimated the bounds on the CSL parameters from the condition $\Delta T_{\mathrm{CSL}}\left(\lambda, r_{C}\right) \geq \Delta T$, where $\Delta T_{\mathrm{CSL}}\left(\lambda, r_{C}\right)$ denotes the theoretical temperature increase predicted by the CSL model.

${ }^{46}$ S. Nimmrichter and K. Hornberger, Phys. Rev. Lett. 110, 160403 (2013). 